\title{
The effect of stress on memory for temporal context: an exploratory study
}

\author{
Nicole D. Montijn, Lotte Gerritsen \& Iris. M. Engelhard \\ Department of Clinical Psychology, Utrecht University, The Netherlands \\ Correspondence to: $\underline{n . d . m o n t i j n @ u u . n l}$
}

\begin{abstract}
Trauma memories can appear dissociated from their original temporal context, and are often relived as they occur in the here-and-now. Potentially these temporal distortions already occur during encoding of the aversive experience as a consequence of stress. Here, 86 participants were subjected to either a stress or control induction, after which they learned the temporal structure of four virtual days. In these virtual days, time was scaled and participants could use clock cues to construe the passage of time within a day. We examined whether stress causes a shift in the learning strategy from one based on virtual time to one based on event sequence. Our results do not show a discernible impact of stress on memory for temporal context, in terms of both sequence memory and more fine-grained representations of time. The stress groups showed more extreme performance trajectories, either good or poor, across all measures. However, as time estimations were overall quite poor it is unclear to what extent this reflected a true strategy shift. Future avenues of research that can build on these findings are discussed.
\end{abstract}

\section{INTRODUCTION}

One of the hallmark features of episodic memory is the temporal relationship between events. Temporal context encapsulates when an episodic event occurred in relation to other events, such as event order and time distance between events, thereby providing a framework by which we can mentally organize and cluster events. Accurate retention of this temporal event context ensures that information is interpreted within the appropriate contextual boundaries, and it protects against overgeneralization of memory (Moore \& Zoellner, 2007). This ability may be especially important for memories of highly emotional or stressful situations where context can act as a buffer to prevent prolonged negative impact of the experience (Al Abed et al., 2020).

Indeed, poor contextualization of memory is known to play a role in various mood and anxiety (related) disorders (Ono et al., 2016; Zlomuzica et al., 2014). Most notably, it is a core feature of posttraumatic stress disorder (PTSD). People suffering from PTSD typically experience 
highly vivid and intrusive traumatic memories that appear to be dissociated from time and place (Brewin, 2011; Brewin et al., 2010; Ehlers \& Clark, 2000). As such, trauma memories are often relived as if occurring in the present with a here-and-now quality. It is possible that these temporal distortions already occur during encoding of the aversive experience as a consequence of physiological stress reactivity (Diamond et al., 2007).

Stress influences memory through a complex time-dependent interaction of noradrenalin and glucocorticoids with hippocampal functioning. The hippocampus is, among other functions, considered central for encoding specific temporal relationships between episodic events (Bellmund et al., 2020, 2021; Palombo et al., 2020) and binding episodic events to a temporal context (DuBrow \& Davachi, 2013; Eichenbaum et al., 2007; Reeders et al., 2018). The effect of stress on memory has been studied extensively over the past few decades across multiple areas of memory research such as encoding, retrieval (Schwabe \& Wolf, 2010, 2014; Wolf, 2009), spatial contextualization (Sazma et al., 2019; van Ast et al., 2013), implicit memory (Meyer et al., 2013; Römer et al., 2011), and emotional memory (Joëls et al., 2011; Payne et al., 2006). Different processes (e.g. acquisition, consolidation, retrieval) are affected, depending on whether stress is experienced before, during or after the memory task. Most consistently, previous studies found that acute stress facilitates consolidation and impairs retrieval of information (Roozendaal, 2002). However, despite the fact that the hippocampus has long been associated with temporal context memory, there are no studies that have directly investigated the relationship between stress and temporal contextualization of memory.

While it is still unclear how stress interferes with temporal memory, it was proposed that stress can cause a shift in learning strategy from cognitive (hippocampus bound) to more habitual (striatum bound) stimulus response learning (Schwabe \& Wolf, 2013). This effect had previously been reported in rodents (e.g., Elliott \& Packard, 2008; Wingard \& Packard, 2008), and this approach to stress and memory has received some attention in human research as well. In spatial learning tasks, stress (Schwabe et al., 2007, 2009; Vogel et al., 2017)) and anticipatory anxiety (Goodman et al., 2012) induce a shift in participants' preferred learning strategy from a 'cognitive' relational strategy to one based on stimulus-response associations. In the cognitive strategy, participants use the spatial configuration of the surrounding area to find a target, while for the stimulus-response strategy they tend to rely on salient landmarks to find the target. Stress seems to affect retrieval strategy in the same way (Zerbes et al., 2020). This effect has been attributed to increased functional connectivity between the amygdala and the striatum as a result of stress-induced glucocorticoid activity (Schwabe et al., 2010; Vogel et al., 2017). Where the hippocampus is involved in forming higher-order representations of event sequences and 
temporal distance between episodic events (Bellmund et al., 2021; Howard, 2017), striatum activity has been associated with implicit learning of sequence information (Rieckmann et al., 2010; Schendan et al., 2003). Thus, it could be that as a result of a stress-induced shift from hippocampal to striatal learning, memory for the order of the event sequence may be relatively preserved while fine-grained temporal information, such as the specific event time and the time distance between events in the sequence, is lost.

Thus far, little is known about the potential origin of the temporal distortion and decontextualization of episodic memories that is often witnessed in stress-related disorders like PTSD. Here, we investigated the time-dependent effect of stress on encoding the temporal structure of episodic event sequences using a 2-day paradigm. Participants were trained to learn the temporal structure of four virtual days either directly after stress/control induction or at a 30minute delay. The next day we assessed their memory for the temporal structure of the virtual days. In these virtual days, time was scaled and participants could use clock cues to construe the passage of time within a day. We examined whether stress causes a shift in the preferred learning strategy from one based on virtual time to a sequence based strategy. We expected that both stress groups would rely more on sequence learning than on virtual time learning, while the control groups would retain a detailed representation of temporal structure (i.e. event time and inter-event time distance) next to the sequential structure as was found in a previous study using this task (Bellmund et al., 2021). The shift in learning strategy has been linked to glucocorticoid activity. Therefore, we expected this effect to be stronger for the 30-min wait stress group compared to the no wait stress group since stress-induced glucocorticoid activity peaks at a delay of about 20 to 30 minutes after the stressor (Schwabe \& Wolf, 2014).

\section{METHODS}

\section{Participants}

Participants were 86 adults ( 40 female, $M$ age $=22.5$, range $=18-32$ ) with no selfreported current psychiatric disorders. They were recruited on campus using flyers, as well as through social media. Female participants were required to be on hormonal birth control. Participants were randomly assigned to one of four experimental groups; stress no wait, stress 30-min wait, control no wait, control 30-min wait (Table 1). All participants provided written informed consent. A power analysis (G*Power Version 3.1; Faul, Erdfelder, Buchner, \& Lang, 2009) based on prior research (Schwabe \& Wolf, 2010) showed that a sample size of at least 20 per group was necessary to detect an effect of stress and timing on memory encoding (power = $\left..90, \eta_{p}{ }^{2}=0,12\right)$. Taking potential missing values into account, we aimed to test $20-25$ participants 
per group. They were remunerated with course credit or had the chance to win a gift card for their participation. The study was approved by the institutional ethical review board at Utrecht University (FETC16-090).

Table 1. Demographic information per group

\begin{tabular}{r|cccc}
\hline \multicolumn{1}{r}{ Condition } & Mage (SD) & Female & Male & Total \\
\hline Stress - no wait & $21.3(1.5)$ & 11 & 9 & 20 \\
Control - no wait & $21.9(2.6)$ & 11 & 14 & 25 \\
Stress - wait & $23.4(2.7)$ & 10 & 10 & 20 \\
Control - wait & $23.4(3.7)$ & 8 & 13 & 21 \\
\hline
\end{tabular}

Study Overview. The experiment was conducted on two consecutive days (Figure 1A), and had a 2 (stress/control) $\times 2$ (wait/no wait) between-subject design. The first day consisted of a stress or control induction followed by a subjective stress questionnaire and an episodic learning task. The first session always took place between 12:30 and 18:00 to control for circadian cortisol rhythms. Participants either started the episodic learning task directly after the stress/control induction, or they had to wait 30 minutes in between tasks, depending on the experimental group. Participants returned the next day for an unannounced memory test about the episodic learning task. The episodic learning task was slightly adapted from Bellmund et al. (2021), and has proved to be effective in teaching participants a novel time scale and temporal structure of episodic material. Compared to the original design, we reduced the number of trials per virtual day from 7 to 4 , and the memory test was conducted a day later rather than shortly after learning. These changes were made to accommodate the task to the stress paradigm in terms of duration and to prevent potential effects of the stress induction on recall rather than just learning. 


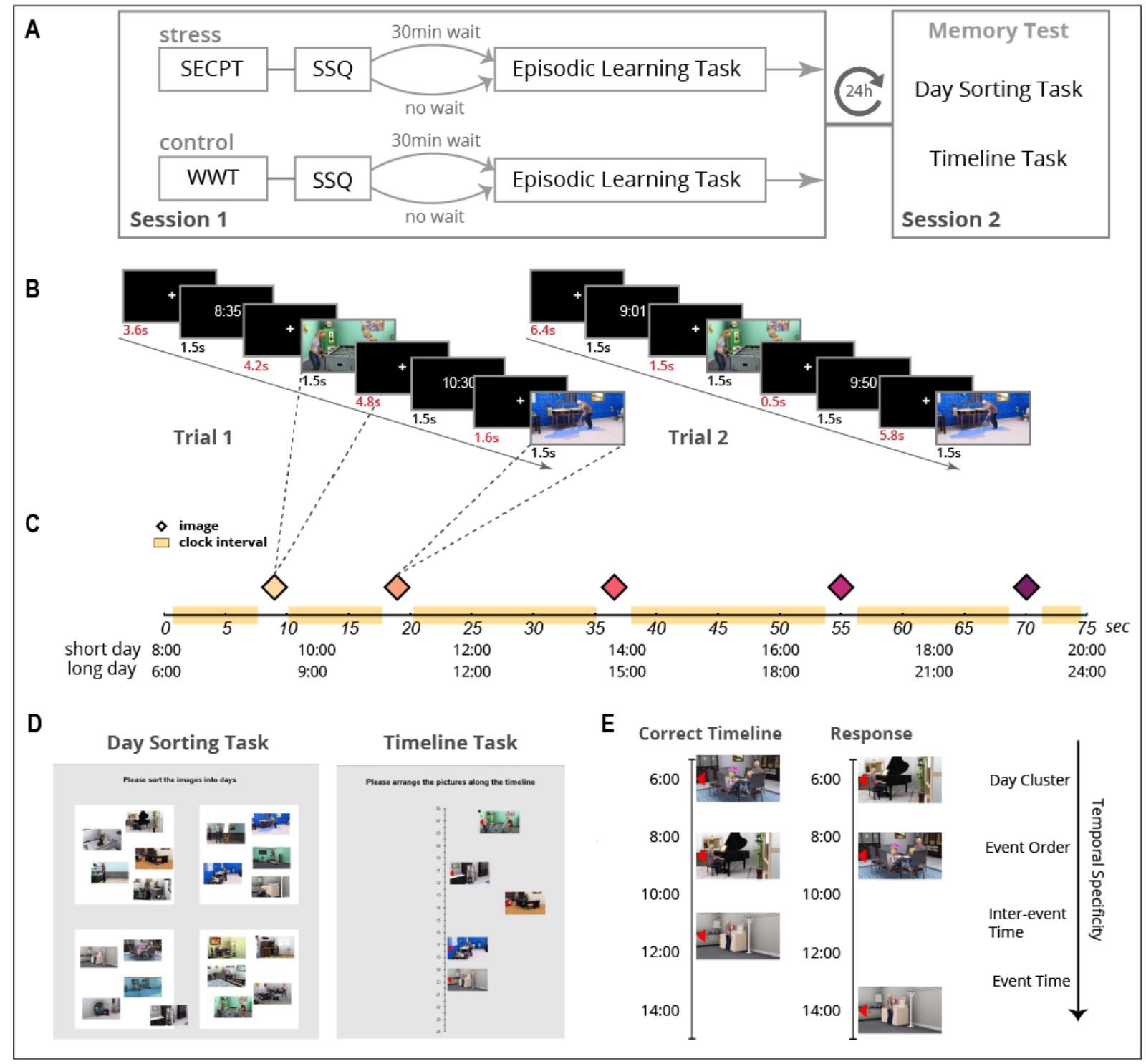

Figure 1. Experimental Design. A) Overview of study procedure for four experimental groups, over two sessions. B) Example of the trial structure of a virtual day, and the difference between two presentations of the same virtual day. Images always take place at the same time within the trial, but clock slides do not and can indicate different times between repetitions of the same virtual day. This allows participants to gradually get a closer estimation of the correct image time. $\mathbf{C}$ ) Illustration of how the time scaling affects the virtual time distance between images for a short and long day with an identical structure. While the time in seconds between images is identical for both the long and short day, the virtual time difference is larger for the long day. Diamonds indicate when an image is shown, and yellow blocks indicate the interval in which a clock will appear. D) Display of both memory tasks. E) Example of a response on the timeline task, and how temporal memory can be abstracted at different levels of specificity. B-E) The Sims 3 and screenshots of it are licensed property of Electronic Arts, Inc. 


\section{Socially Evaluated Cold Pressor Test}

Stress induction. The socially evaluated cold pressor test (hereafter SECPT; Schwabe et al., 2008) was used to induce physiological and social stress. Participants were instructed to submerge their non-dominant hand in a bucket of ice-cold water (0-2 degrees Celsius), for a maximum duration of 3 minutes. They were told that they could remove their hand from the water if it became unbearable, but to keep it in as long as they could. As a social stressor, the test leader wore a lab coat and took on a strict and highly formal demeanor. During the task, the test leader actively monitored participants while tracking the time using a stopwatch. Additionally, participants were told that their facial expressions would be recorded with a desk camera. After 3 minutes, or earlier when participants removed their hand from the water, they were given a towel to dry their hand and were asked to complete a short subjective stress questionnaire (SSQ). The SSQ asked them to rate how stressful, painful, and unpleasant they found the task on a 10point Likert scale $(0-100)$. Participants either proceeded directly to the episodic learning task or were asked to wait 30 minutes while reading unstimulating magazines (e.g. home improvement or sailing magazines). They were not allowed to use their phone.

Control induction. The control conditions followed the same procedure as the stress conditions. However, the SECPT was replaced by the Warm Water Test (hereafter WWT; Schwabe et al., 2008). The WWT has the same general structure as the SECPT, but the water is lukewarm (20-23 degrees Celsius) and none of the social stressors are applied. The test leader stayed in the room with the participant during the task to track time, but did not actively monitor participants and stayed outside of their line of sight.

\section{Materials and Episodic Learning Task}

Scene images. We used 20 images, created using the life simulation video game The Sims 3 (The Sims 3 and screenshots of it are licensed property of Electronic Arts, Inc.), to construct virtual days for the episodic learning task. The images displayed everyday activities in the life of a Sim family (e.g. reading the newspaper or doing homework). All images depicted a unique scene, and they were independently rated by a sample of 40 students (Bellmund et al., 2021) as visually distinct, temporally ambiguous, and clear in terms of content. Temporal ambiguity was of particular importance as each of the 20 images was randomly assigned to one of five fixed time-points within one of 4 virtual days.

Virtual days. Each of the four virtual days consisted of 5 images that were assigned to specific time-points within the virtual day to create a 4 unique event sequences. The time-points at which an image took place were identical for all participants. Participants' task was to 
memorize which 5 images belonged to the same day, and to estimate at what time within the virtual day each image took place.

Within the virtual days, the passage of time was scaled so that each second represented a certain number of minutes in virtual time. There were two short days, 8:00 - 20:00, and two long days, 6:00 - 0:00. The short and long days had the same duration in real time $(75 \mathrm{sec})$ but differed in virtual time (12h and 18h). Thus, time passed more quickly in long days ( $1 \mathrm{sec}=14,4 \mathrm{~min})$ than short days (1 sec = 9,6 $\mathrm{min}$ ). In order to correctly estimate the time-point of each image, participants had to learn these time scales. The different time-scales allow dissociation between memory based on virtual time and real time. They were implemented in an earlier neuro-imaging study using the same task (Bellmund et al., 2021).

Clocks. To enable participants to estimate virtual time, a clock with the current virtual time was shown before and after each image (Figure 1B and C). Clocks appeared once at a random moment within specified intervals (yellow bars in Figure 1C). They never appeared within the 15 virtual minutes before or after an image was displayed. Crucially, clocks never appeared at the same moment across repetitions of the same day (Figure 1B). This allowed participants to get a closer estimate of the correct time with every repetition of a virtual day. The goal of this procedure was for participants to develop a sense of (virtual) time without relying on direct associations between clock time and an image.

Episodic Learning Task. Participants were tasked with learning the temporal structure of the 4 virtual days. They were informed that each of the images occurred at a specific time within a virtual day, and that their task was to learn both which images belonged to the same day and at what time they took place using clocks. Each virtual day was presented 4 times. The episodic learning task consisted of 4 blocks of 4 trials, making a total of 16 trials. Each virtual day was presented once per block. An image of a moon was shown at the end of each virtual day to allow participants to differentiate between the four days within each block. The order of the four days was randomized within blocks, and there was a 30 second break between blocks. The task took about 20 minutes to complete.

Memory test. One day later participants returned to the lab for an unannounced memory test. They were told that they would do a similar task as in the first session, but the exact content was not disclosed. The memory test consisted of two parts (Figure 1D). In the first part, participants had to recall which images belonged to the same virtual day. The 20 images appeared on the screen in a circular formation, in randomized order. Participants could drag and drop each image into one of four white squares that represented the four days. The virtual days were not given explicit labels during the learning task. So participants simply grouped the five 
images they thought belonged to the same day in one of the squares. This task assesses the ability to recall the larger temporal context to which single events belong.

In the second part, participants were asked to reproduce at which time each image took place within the virtual day by placing it along a timeline. The four virtual days were presented separately. A timeline was presented on screen that ran from 6:00 till 0:00, as well as the 5 images that belonged to that day. A red arrow was embedded in each image to allow more precise placement along the timeline. When participants were finished with a day they could continue with the next day. This task assesses more fine-grained temporal context memory, namely memory for event sequence, time distance between events in a sequence, and time of occurrence. The memory test took about 10 to 15 minutes to complete.

\section{Statistical analysis}

Subjective stress levels. To determine if the stress induction was successful and similar for both stress groups, we compared SSQ scores of the four experimental groups using a 2 (Condition: control, stress) $\times 2$ (Wait: wait, no wait) between subjects ANOVA.

Memory test. For the day sorting task, we first had to identify which square represented which virtual day. To do so, we compared participants' responses to the correct image placement for all potential day orders (e.g. 1;3;2;4 or 3;2;1;4 etc.). We selected the day order that best matched the participant's response. Given the selected day order, we tallied how many images were correctly placed in each square. So if the top left square represented day 2 and contained three images of day 2 , then the square received a score of 3 . The total sorting score had a maximum of 20 and a minimum of 7.

Performance on the timeline task was operationalized in three time metrics: sequential distance, virtual time distance, and inter-event time distance (Figure 1E). Scores for all three metrics reflected the absolute deviation from the correct response expressed as order (e.g. 2 positions off) or virtual time distance. The virtual time distance was converted from virtual minutes to seconds in real time (short days: VT / 9.6 * 60, long days: VT / 14.4 * 60) to correct for the different timescales of the long and short days (see 'Virtual days' above). For the three time metrics, an average score of 0 reflects perfect performance. This caused the response distribution to be skewed towards 0 . Therefore, Mann-Whitney $U$ tests were used to investigate the effect of stress induction and wait time on temporal learning. 


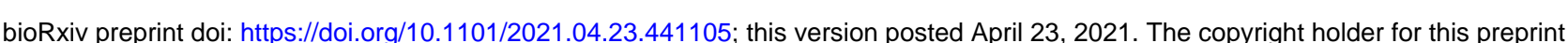
(which was not certified by peer review) is the author/funder, who has granted bioRxiv a license to display the preprint in perpetuity. It is made available under aCC-BY-NC 4.0 International license.

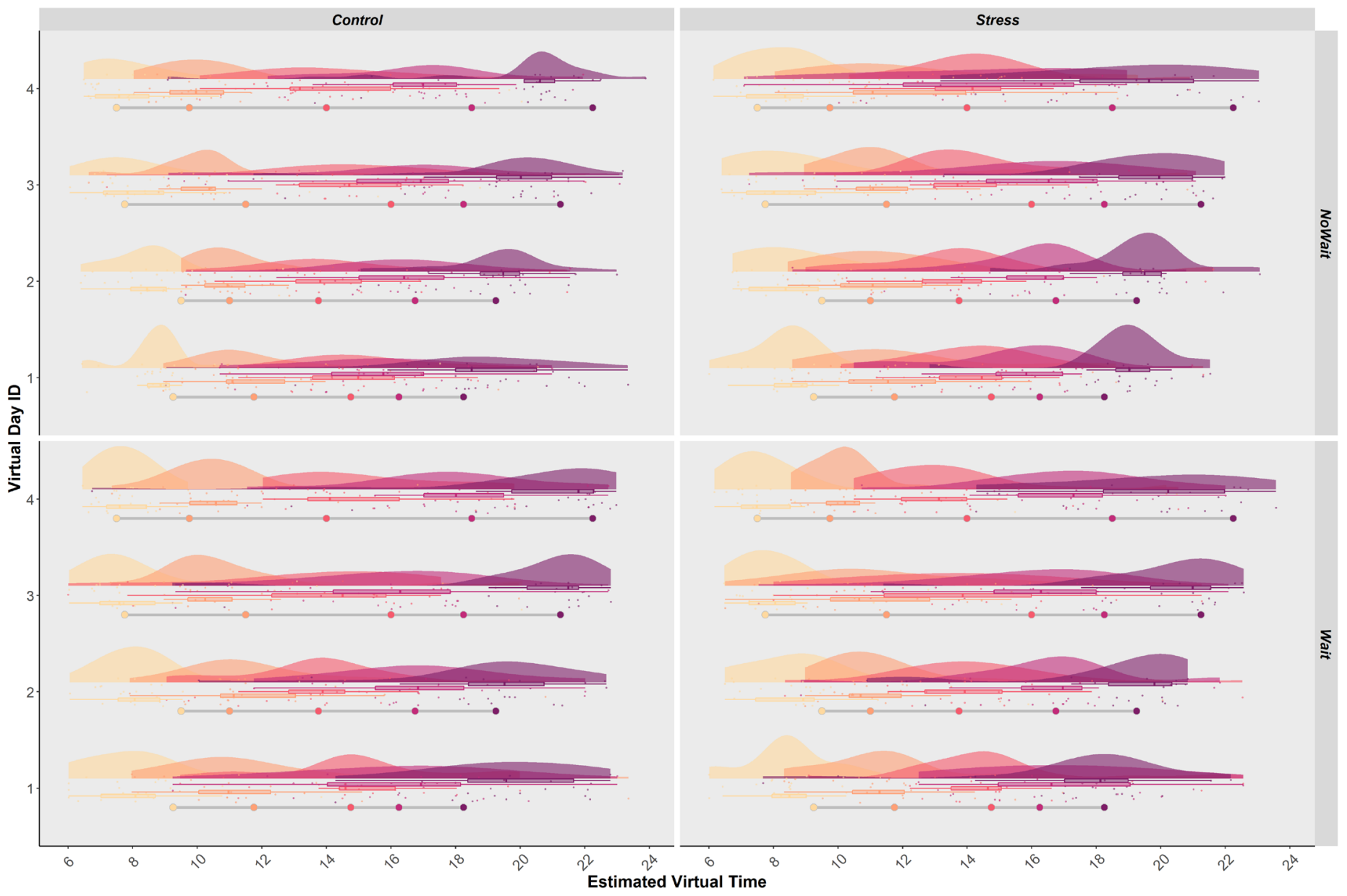

Figure 2. Response distribution of the estimated virtual time of each event image in a virtual day, separated by experimental group. The correct event timeline is shown in grey beneath each response distribution, colored circles represent each event image.

\section{RESULTS}

Subjective Stress. We first verified whether the stress induction was successful by comparing group differences in self-reported subjective stress using a 2 (Condition: control, stress) $\times 2$ (Wait: wait, no wait) between subjects ANOVA. We found a main effect of Condition, $F(1,82)=286.14, p<$ $.001, \eta_{p}^{2}=.78$, indicating that the SECPT indeed induced more subjective stress than the control induction (Stress: $M=160.8, S D=59.9$, Control: $M=11.5, S D=18.6$ ). However, there was also a significant interaction between Condition and Wait, $F(1,82)=6.41, p=.013, \eta_{p}^{2}=.073$. This interaction was due to the Stress - Wait group $(M=183.0, S D=62.7)$ scoring significantly higher on subjective stress than the Stress - No Wait group $(M=138.5, S D=49.0), t(38)=-2.499, p=.017$. This difference in subjective stress limits direct comparisons between the two stress groups. 
bioRxiv preprint doi: https://doi.org/10.1101/2021.04.23.441105; this version posted April 23, 2021. The copyright holder for this preprint (which was not certified by peer review) is the author/funder, who has granted bioRxiv a license to display the preprint in perpetuity. It is

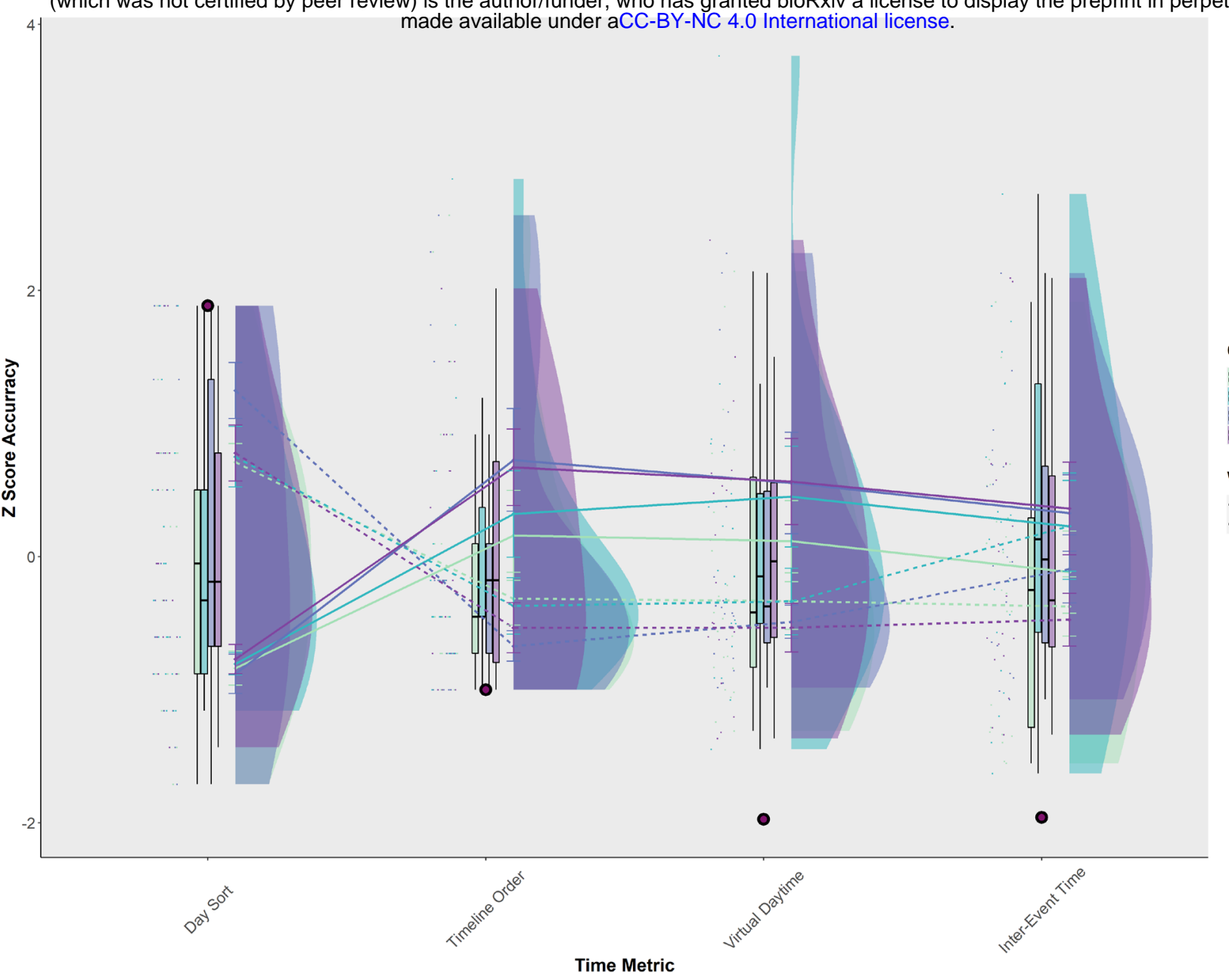

Group

Control NoWait

Control Wait

Stress NoWait

Stress Wait

Within Group Median Split

- Lower

- Upper

Figure 3. Data distribution of Z scores for performance on day sorting, timeline order, virtual time estimation and inter-event virtual time estimation. Black dots represent the Z-score that indicates perfect performance on each measure (i.e. score of 20 on day sorting, and 0 on the three Timeline measures). Lines represent the group means on each measure of high (dashed line) and low (full line) performers within each group. High and low performers were categorized based on a within group median split of the on the day sorting task scores.

Day Sorting Task. A Mann-Whitney $U$ test was used to examine the effect of stress on encoding of episodic context, as indexed by which event images belong to the same virtual day. We did not find a significant difference in performance between the stress and control group in both the No Wait (Control: $M(S D)=12.84$ (3.3); Stress: $M(S D)=13.85(4.4)), U=216.5, p=.442$, and Wait condition (Control: M (SD)= 12.95 (3.4); Stress: M (SD)= $13.20(3.4)), U=195, p=.694$. Furthermore, there was no difference in performance between the stress - wait and stress - no wait groups, $U=184.5, p=.678$.

Timeline Task. First, we compared the Stress groups with their respective Control group (e.g. Stress wait with control wait) to examine the effect of stress on memory for temporal structure. No significant differences were found between the stress and control groups for any of the three time metrics (No Wait: all $U>192, p>$.185; Wait: all $U>177, p>$.389). Second, we compared the Stress groups to examine the effect of the interval between stress and learning on 
memory for temporal structure. Again, no group differences were found on any of the measures (all $U>178, p>.552$ ).

Bayesian. Bayesian independent-sample t-tests were performed in JASP (JASP Version 0.14.01) to assess evidence for the null hypothesis (i.e. no difference between the stress and control groups). A default Cauchy prior of 0.707 was used as it was difficult to determine an informed prior. Comparing the stress no wait and the control no wait group, we found anecdotal to moderate evidence for the null hypothesis across all outcome measures: Day Sorting $\mathrm{B}_{10}=$ $.403,95 \% \mathrm{Cl}[-.756, .314]$, Event Sequence $\mathrm{B}_{10}=.307,95 \% \mathrm{Cl}[-.603, .451]$, Time Deviation $\mathrm{B}_{10}=$ $.321,95 \% \mathrm{Cl}[-.641, .416]$, Inter-Event Time $\mathrm{B}_{10}=.584,95 \% \mathrm{Cl}[-.877, .215]$. Comparing the stress wait and the control wait group, we again found anecdotal to moderate evidence for the null hypothesis across all outcome measures: Day Sorting $\mathrm{B}_{10}=.313,95 \% \mathrm{Cl}[-.605, .485]$, Event Sequence $\mathrm{B}_{10}=.314,95 \% \mathrm{Cl}[-.612, .478]$, Time Deviation $\mathrm{B}_{10}=.31,95 \% \mathrm{Cl}[-.496, .593]$, Inter-Event Time $\mathrm{B}_{10}=.406,95 \% \mathrm{Cl}[-.334, .774]$.

Performance trajectory. Previous research on the stress induced shift in learning strategy highlights that this shift does not occur ubiquitously, but rather the ratio of individuals in a group that use a stimulus-response based strategy goes up in response to stress. Therefore, because evidence for the null hypothesis was limited, we decided to further explore individual performance trajectories across the four measures going down in temporal specificity (Figure 1E). We divided the four experimental groups into high and low performers based on a within group median split on the day sorting scores. Subsequently, we examined the performance trajectory of the high and low scoring groups on the three time metrics. Within the stress groups, differences between high and low performers were more extreme across all measures, while these differences were less pronounced or not present in the control group (see Supplementary Table 1). This seems to indicate that under the influence of stress accurate memory for episodic context promotes the retention of more detailed temporal information like sequence order and event time, while it worsens memory for such information if the episodic context is not retained.

\section{DISCUSSION}

This study aimed to provide a first look at the effect of physiological stress on encoding temporal context in an episodic memory task. We expected that stress would cause participants to rely more on item-item associations (sequence order) to deduce temporal context rather than the specific time of occurrence (virtual time deviation). Contrary to our expectations, our results do not show a pronounced effect of stress on memory for temporal context. Compared to controls, the stress groups did show more extreme response patterns, performing either very well or very 
poorly across all measures depending on their memory of the larger episodic context (i.e. the virtual days). In earlier work, a strategy shift was observable as the relative percentage of a group that utilized item-item associations (i.e. sequence order) over the cognitive strategy (i.e. virtual time). However, due to the large inter-individual differences in the ability to estimate virtual time that we observed independent of stress, it is unclear to what extent a true strategy shift occurred within the stress groups. Both the control and stress groups scored quite poorly on the virtual time measures (mean error of about 1,5-2,5 virtual hours), which may have obscured a stress induced strategy shift. Performance was best for the event sequence metric across groups, which seems to indicate that participants generally focused on learning the event sequence rather than the virtual time and still required a strong cue (i.e. being given the correct items per day) to access that knowledge.

It is possible that the effect of stress on temporal context memory depends on the presence of emotionally arousing or stress relevant stimuli. The current task was not intrinsically related to the stressor and did not include emotional material, which could have reduced a potential effect of stress. While the learning strategy shift is thought to occur independently of emotional content, a large body of work shows that emotional arousal is a boundary condition for stress to affect memory (Buchanan \& Tranel, 2008; Joëls et al., 2011; van Ast et al., 2013; Wolf, 2008). The physiological stress reaction generally biases attention towards information related to the stressor, which is innately emotionally arousing. This attentional bias is known to disrupt encoding of information that is deemed less relevant in the current situation in favor of information that promotes immediate survival. An exciting example of how a temporal learning task can act as the stressor is a recent study that examined temporal clustering in memory by having participants walk through a haunted house (Gregory, 2020; Reisman et al., 2021). These types of "one-shot" learning paradigms more closely approximate the manner in which trauma memories are acquired than the current task, and could offer a naturalistic yet controlled way of examining the effect of stress on memory.

A further explanation for the lack of a pronounced stress effect is task related. A previous study that used the current paradigm found that event time estimates on the timeline task are systematically biased towards the mean virtual time of events that hold the same sequential position on the other three virtual days (Bellmund et al., 2021). Furthermore, events from separate days had increased hippocampal neural pattern similarity if the events were close together in time, and decreased pattern similarity if they were further apart. Bellmund et al. (2021) suggest that this effect reflects the role the hippocampal-entorhinal region takes in abstracting regularity, or semantic information, from episodic events. This generalization effect 
was also found in the current dataset (data from our control groups are presented as part of Bellmund et al., 2021). Because of this unexpectedly strong generalization effect, potentially, amplified through enhanced task difficulty, the memory task became less sensitive to differences in memory for virtual time between groups as responses regressed towards a mean timeline. Subsequent studies using this paradigm may prevent this issue by increasing the amount trials in the learning task, or by assessing memory after a shorter interval.

While these two factors (i.e. non-emotional learning task and generalization effect) may explain the absence of a stress effect here, another potential explanation is that the temporal distortions seen in memories of trauma do not stem from the physiological stress reaction during encoding but rather occur retroactively. Although memories of traumatic events are often remembered vividly, these memories are subject to change over time (e.g., Engelhard \& McNally, 2015). Repeated involuntary recollection or re-experiencing symptoms, which are common after trauma, can facilitate recall (García-Bajos \& Migueles, 2017) but can also introduce memory errors (Thomas et al., 2003). Similar to our finding that memory for event time in this task is biased by knowledge of structurally similar event sequences, episodic memories can generalize over time in favor of the abstraction of structural semantic knowledge (Moscovitch et al., 2016; Renoult et al., 2019). Thereby, selective rehearsal of core elements of the trauma memory may gradually make the broader (less rehearsed) spatiotemporal event context less accessible, giving the resulting memory a more semantic (atemporal) quality.

A limitation of the current study is the lack of an objective measure of stress reactivity. The shift to striatal learning we expected to find here is thought to critically depend on glucocorticoid activity, that modulates the functional connectivity between the amygdala and the striatum (Schwabe et al., 2010; Vogel et al., 2017). Thus, it is likely that glucocorticoid levels moderate the shift towards striatal learning strategies. Both subjective stress and cortisol responses are known to vary widely between people due to factors like sex, age, menstrual cycle and chronic stress (Kirschbaum et al., 1999; Kudielka et al., 2009). In addition, subjective stress and physiological stress reactivity do not always correlate well (Campbell \& Ehlert, 2012; Duchesne \& Pruessner, 2013). Therefore, future work should consider including salivary cortisol as a measure of stress reactivity next to subjective stress measurements. Indeed, one study demonstrated that endogenous cortisol secretion moderated the impairing effect of stress on implicit spatial learning (Meyer et al., 2011). Specifically, high cortisol responders improved compared to controls whereas low responders performed worse. While this finding contradicts the effect generally observed in explicit learning tasks, it does show how cortisol reactivity can lead to opposing performance trajectories such as observed in the current sample. 
In summary, this study constitutes a first attempt at disseminating the effect of stress on encoding of temporal context information in episodic memory. The current results do not show a discernible impact of stress on the ability to remember temporal context, in terms of both sequence memory and more fine-grained representations of time. However, several limitations of the current design may have reduced task sensitivity. Specifically, the generalization of memory to an average timeline harkens back the observed overgeneralization of memory in stress and trauma related disorders (Brown et al., 2014; McNally et al., 1995), and should be explored further in future work.

Acknowledgements. We would like to thank Christian Doeller and Lorena Deuker for permitting us to use the episodic learning task that was originally developed by Lorena Deuker and NDM as part of Bellmund et al. (2021). We thank Maja Kalkofen and Vanessa Danzer for their assistance with data collection.

Author Contributions. NDM, LG and IME designed the study; NDM collected the data; NDM and LG analyzed the data; all authors interpreted the data; NDM wrote the first draft of the article, and LG and IME provided critical revisions. All authors approved the final version of the manuscript for submission.

Funding. This study was supported with a Vici grant from the Netherlands Organization for Scientific Research (NWO 453-15-005) awarded to IME.

Declaration of Conflicting Interests. The author(s) declared that there were no conflicts of interest with respect to the authorship or the publication of this article. 
bioRxiv preprint doi: https://doi.org/10.1101/2021.04.23.441105; this version posted April 23, 2021. The copyright holder for this preprint (which was not certified by peer review) is the author/funder, who has granted bioRxiv a license to display the preprint in perpetuity. It is made available under aCC-BY-NC 4.0 International license.

\section{SUPPLEMENTAL TABLES}

Supplementary Table 1. Mean and SD of performance on timeline order, virtual time estimation, and interevent time estimation for high and low performers on the day sorting task in the Stress and Control groups. On all measures, a score closer to 0 reflects better performance.

\begin{tabular}{|c|c|c|c|c|c|c|}
\hline \multirow[t]{2}{*}{ Time Metric } & \multicolumn{2}{|c|}{ Control } & \multicolumn{4}{|c|}{ Stress } \\
\hline & High & Low & Mann-Whitney U & High & Low & Mann-Whitney U \\
\hline Timeline Order & $\begin{array}{l}M=.24 \\
S D=.24\end{array}$ & $\begin{array}{l}M=.45 \\
S D=.41\end{array}$ & $U=175, p=0.047$ * & $\begin{array}{l}M=.15 \\
S D=.17\end{array}$ & $\begin{array}{c}M=.62 \\
S D=.38\end{array}$ & $U=50, p<.001$ ** \\
\hline $\begin{array}{l}\text { Virtual Time } \\
\text { Deviation }\end{array}$ & $\begin{array}{c}M=8.12 \\
S D= \\
3.67\end{array}$ & $\begin{array}{l}M=11.11 \\
S D=5.73\end{array}$ & $U=178, p=0.059$ & $\begin{array}{l}M=7.25 \\
S D=2.38\end{array}$ & $\begin{array}{l}M=12.55 \\
S D=5.38\end{array}$ & $U=74, p<.001 * *$ \\
\hline $\begin{array}{l}\text { Inter-Event time } \\
\text { deviation }\end{array}$ & $\begin{array}{c}M=4.89 \\
S D= \\
2.51\end{array}$ & $\begin{array}{l}M=5.26 \\
S D=3.15\end{array}$ & $U=258, p=.895$ & $\begin{array}{c}M=4.41 \\
S D=1.92\end{array}$ & $\begin{array}{l}M=6.05 \\
S D=2.58\end{array}$ & $U=121, p=.033$ * \\
\hline
\end{tabular}




\section{REFERENCES}

Al Abed, A. S., Ducourneau, E.-G., Bouarab, C., Sellami, A., Marighetto, A., \& Desmedt, A. (2020). Preventing and treating PTSD-like memory by trauma contextualization. Nature Communications, 11(1), 4220. https://doi.org/10.1038/s41467-020-18002-w

Bellmund, J. L. S., Deuker, L., Montijn, N. D., \& Doeller, C. F. (2021). Structuring time: The hippocampus constructs sequence memories that generalize temporal relations across experiences. bioRxiv.

Bellmund, J. L. S., Polti, I., \& Doeller, C. F. (2020). Sequence Memory in the HippocampalEntorhinal Region. Journal of Cognitive Neuroscience, 32(11), 2056-2070. https://doi.org/10.1162/jocn_a_01592

Brewin, C. R. (2011). The nature and significance of memory disturbance in posttraumatic stress disorder. Annual Review of Clinical Psychology, 7, 203-227. https://doi.org/10.1146/annurev-clinpsy-032210-104544

Brewin, C. R., Gregory, J. D., Lipton, M., \& Burgess, N. (2010). Intrusive images in psychological disorders: characteristics, neural mechanisms, and treatment implications. Psychological Review, 117(1), 210-232. https://doi.org/10.1037/a0018113

Brown, A. D., Addis, D. R., Romano, T. A., Marmar, C. R., Bryant, R. A., Hirst, W., \& Schacter, D. L. (2014). Episodic and semantic components of autobiographical memories and imagined future events in post-traumatic stress disorder. Memory, 22(6), 595-604. https://doi.org/10.1080/09658211.2013.807842

Buchanan, T. W., \& Tranel, D. (2008). Stress and emotional memory retrieval: effects of sex and cortisol response. Neurobiology of Learning and Memory, 89(2), 134-141. https://doi.org/10.1016/j.nlm.2007.07.003

Campbell, J., \& Ehlert, U. (2012). Acute psychosocial stress: does the emotional stress response correspond with physiological responses? Psychoneuroendocrinology, 37(8), 1111-1134. https://doi.org/10.1016/j.psyneuen.2011.12.010

Diamond, D. M., Campbell, A. M., Park, C. R., Halonen, J., \& Zoladz, P. R. (2007). The temporal dynamics model of emotional memory processing: a synthesis on the neurobiological basis of stress-induced amnesia, flashbulb and traumatic memories, and the YerkesDodson law. Neural plasticity, 2007, 60803. https://doi.org/10.1155/2007/60803

DuBrow, S., \& Davachi, L. (2013). The influence of context boundaries on memory for the sequential order of events. Journal of Experimental Psychology: General, 142(4), 1277-1286. https://doi.org/10.1037/a0034024 
Duchesne, A., \& Pruessner, J. C. (2013). Association between subjective and cortisol stress response depends on the menstrual cycle phase. Psychoneuroendocrinology, 38(12), 31553159. https://doi.org/10.1016/j.psyneuen.2013.08.009

Ehlers, A., \& Clark, D. M. (2000). A cognitive model of posttraumatic stress disorder. Behaviour Research and Therapy, 38(4), 319-345. https://doi.org/10.1016/s0005-7967(99)00123-0

Eichenbaum, H., Yonelinas, A. P., \& Ranganath, C. (2007). The medial temporal lobe and recognition memory. Annual Review of Neuroscience, 30, 123-152. https://doi.org/10.1146/annurev.neuro.30.051606.094328

Elliott, A. E., \& Packard, M. G. (2008). Intra-amygdala anxiogenic drug infusion prior to retrieval biases rats towards the use of habit memory. Neurobiology of Learning and Memory, 90(4), 616-623. https://doi.org/10.1016/j.nlm.2008.06.012

Engelhard, I. M., \& McNally, R. J. (2015). Metacognitive appraisal of memory inconsistency for traumatic events in Dutch veterans. Memory, 23(7), 972-980. https://doi.org/10.1080/09658211.2014.942669

García-Bajos, E., \& Migueles, M. (2017). Retrieval of past and future positive and negative autobiographical experiences. Cognition \& emotion, 31(6), 1260-1267. https://doi.org/10.1080/02699931.2016.1204988

Goodman, J., Leong, K.-C., \& Packard, M. G. (2012). Emotional modulation of multiple memory systems: implications for the neurobiology of post-traumatic stress disorder. Reviews in the neurosciences, 23(5-6), 627-643. https://doi.org/10.1515/revneuro-2012-0049

Gregory, D. (2020, August 18). Disruption of temporal clustering and forward transition movement during memory recall for a real-world, emotionally-arousing event [Poster Presentation]. Context and Episodic Memory Symposium Virtual Meeting, Philadelphia, Pennsylvania, United States.

Howard, M. W. (2017). Temporal and spatial context in the mind and brain. Current Opinion in Behavioral Sciences, 17, 14-19. https://doi.org/10.1016/j.cobeha.2017.05.022

Joëls, M., Fernandez, G., \& Roozendaal, B. (2011). Stress and emotional memory: a matter of timing. Trends in Cognitive Sciences, 15(6), 280-288. https://doi.org/10.1016/j.tics.2011.04.004

Kirschbaum, C., Kudielka, B. M., Gaab, J., Schommer, N. C., \& Hellhammer, D. H. (1999). Impact of gender, menstrual cycle phase, and oral contraceptives on the activity of the hypothalamus-pituitary-adrenal axis. Psychosomatic Medicine, 61(2), 154-162. https://doi.org/10.1097/00006842-199903000-00006 
Kudielka, B. M., Hellhammer, D. H., \& Wüst, S. (2009). Why do we respond so differently? Reviewing determinants of human salivary cortisol responses to challenge. Psychoneuroendocrinology, 34(1), 2-18. https://doi.org/10.1016/j.psyneuen.2008.10.004

McNally, R. J., Lasko, N. B., Macklin, M. L., \& Pitman, R. K. (1995). Autobiographical memory disturbance in combat-related posttraumatic stress disorder. Behaviour Research and Therapy, 33(6), 619-630. https://doi.org/10.1016/0005-7967(95)00007-k

Meyer, T., Smeets, T., Giesbrecht, T., \& Merckelbach, H. (2011). Individual differences in hippocampal configuration learning as predictor of intrusive memories. Neuroscience Letters, 500, e41. https://doi.org/10.1016/j.neulet.2011.05.187

Meyer, T., Smeets, T., Giesbrecht, T., Quaedflieg, C. W. E. M., \& Merckelbach, H. (2013). Acute stress differentially affects spatial configuration learning in high and low cortisolresponding healthy adults. European journal of psychotraumatology, 4. https://doi.org/10.3402/ejpt.v4i0.19854

Moore, S. A., \& Zoellner, L. A. (2007). Overgeneral autobiographical memory and traumatic events: an evaluative review. Psychological Bulletin, 133(3), 419-437. https://doi.org/10.1037/0033-2909.133.3.419

Moscovitch, M., Cabeza, R., Winocur, G., \& Nadel, L. (2016). Episodic memory and beyond: the hippocampus and neocortex in transformation. Annual review of psychology, 67(1), 105134. https://doi.org/10.1146/annurev-psych-113011-143733

Ono, M., Devilly, G. J., \& Shum, D. H. K. (2016). A meta-analytic review of overgeneral memory: The role of trauma history, mood, and the presence of posttraumatic stress disorder. Psychological trauma: theory, research, practice and policy, 8(2), 157-164. https://doi.org/10.1037/tra0000027

Palombo, D. J., Reid, A. G., Thavabalasingam, S., Hunsberger, R., Lee, A. C. H., \& Verfaellie, M. (2020). The Human Medial Temporal Lobe Is Necessary for Remembering Durations within a Sequence of Events but Not Durations of Individual Events. Journal of Cognitive Neuroscience, 32(3), 497-507. https://doi.org/10.1162/jocn_a_01489

Payne, J. D., Jackson, E. D., Ryan, L., Hoscheidt, S., Jacobs, J. W., \& Nadel, L. (2006). The impact of stress on neutral and emotional aspects of episodic memory. Memory, 14(1), 1-16. https://doi.org/10.1080/09658210500139176

Reeders, P. C., Allen, T. A., \& Mattfeld, A. T. (2018). Hippocampus activations reflect temporal contexts while medial prefrontal cortex activations reflect ordinal positions during sequence memory in humans: Supplemental Figure 1 and Table 1. BioRxiv. https://doi.org/10.1101/501122 
bioRxiv preprint doi: https://doi.org/10 1101/2021.04 23 441105; this version posted April 23, 2021. The copyright holder for this preprint (which was not certified by peer review) is the author/funder, who has granted bioRxiv a license to display the preprint in perpetuity. It is made available under aCC-BY-NC 4.0 International license.

Reisman, S., Gregory, D. F., Stasiak, J., Mitchell, W. J., Helion, C., \& Murty, V. P. (2021). Influence of Naturalistic, Emotional Context and Intolerance of Uncertainty on Arousal-Mediated Biases in Episodic Memory. https://doi.org/10.31234/osf.io/fy2tm

Renoult, L., Irish, M., Moscovitch, M., \& Rugg, M. D. (2019). From Knowing to Remembering: The Semantic-Episodic Distinction. Trends in Cognitive Sciences, 23(12), 1041-1057. https://doi.org/10.1016/j.tics.2019.09.008

Rieckmann, A., Fischer, H., \& Bäckman, L. (2010). Activation in striatum and medial temporal lobe during sequence learning in younger and older adults: relations to performance. Neuroimage, 50(3), 1303-1312. https://doi.org/10.1016/j.neuroimage.2010.01.015

Römer, S., Schulz, A., Richter, S., Lass-Hennemann, J., \& Schächinger, H. (2011). Oral cortisol impairs implicit sequence learning. Psychopharmacology, 215(1), 33-40. https://doi.org/10.1007/s00213-010-2112-4

Roozendaal, B. (2002). Stress and memory: opposing effects of glucocorticoids on memory consolidation and memory retrieval. Neurobiology of Learning and Memory, 78(3), 578-595. https://doi.org/10.1006/nlme.2002.4080

Sazma, M. A., McCullough, A. M., Shields, G. S., \& Yonelinas, A. P. (2019). Using acute stress to improve episodic memory: The critical role of contextual binding. Neurobiology of Learning and Memory, 158, 1-8. https://doi.org/10.1016/j.nlm.2019.01.001

Schendan, H. E., Searl, M. M., Melrose, R. J., \& Stern, C. E. (2003). An FMRI study of the role of the medial temporal lobe in implicit and explicit sequence learning. Neuron, 37(6), 1013-1025. https://doi.org/10.1016/s0896-6273(03)00123-5

Schwabe, L., Haddad, L., \& Schachinger, H. (2008). HPA axis activation by a socially evaluated coldpressor test. Psychoneuroendocrinology, 33(6), 890-895. https://doi.org/10.1016/j.psyneuen.2008.03.001

Schwabe, L., Oitzl, M. S., Philippsen, C., Richter, S., Bohringer, A., Wippich, W., \& Schachinger, H. (2007). Stress modulates the use of spatial versus stimulus-response learning strategies in humans. Learning \& Memory, 14(1), 109-116. https://doi.org/10.1101/Im.435807

Schwabe, L., Oitzl, M. S., Richter, S., \& Schächinger, H. (2009). Modulation of spatial and stimulusresponse learning strategies by exogenous cortisol in healthy young women. Psychoneuroendocrinology, 34(3), 358-366. https://doi.org/10.1016/j.psyneuen.2008.09.018

Schwabe, L., Schächinger, H., de Kloet, E. R., \& Oitzl, M. S. (2010). Corticosteroids operate as a switch between memory systems. Journal of Cognitive Neuroscience, 22(7), 1362-1372. https://doi.org/10.1162/jocn.2009.21278 
Schwabe, L., \& Wolf, O. T. (2010). Learning under stress impairs memory formation. Neurobiology of Learning and Memory, 93(2), 183-188. https://doi.org/10.1016/j.nlm.2009.09.009

Schwabe, L., \& Wolf, O. T. (2013). Stress and multiple memory systems: from "thinking" to "doing". Trends in Cognitive Sciences, 17(2), 60-68. https://doi.org/10.1016/j.tics.2012.12.001

Schwabe, L., \& Wolf, O. T. (2014). Timing matters: temporal dynamics of stress effects on memory retrieval. Cognitive, Affective \& Behavioral Neuroscience, 14(3), 1041-1048. https://doi.org/10.3758/s13415-014-0256-0

Thomas, A. K., Bulevich, J. B., \& Loftus, E. F. (2003). Exploring the role of repetition and sensory elaboration in the imagination inflation effect. Memory \& Cognition, 31(4), 630-640. https://doi.org/10.3758/bf03196103

van Ast, V. A., Cornelisse, S., Meeter, M., Joëls, M., \& Kindt, M. (2013). Time-dependent effects of cortisol on the contextualization of emotional memories. Biological Psychiatry, 74(11), 809816. https://doi.org/10.1016/j.biopsych.2013.06.022

Vogel, S., Klumpers, F., Schröder, T. N., Oplaat, K. T., Krugers, H. J., Oitzl, M. S., Joëls, M., Doeller, C. F., \& Fernández, G. (2017). Stress Induces a Shift Towards Striatum-Dependent StimulusResponse Learning via the Mineralocorticoid Receptor. Neuropsychopharmacology, 42(6), 1262-1271. https://doi.org/10.1038/npp.2016.262

Wingard, J. C., \& Packard, M. G. (2008). The amygdala and emotional modulation of competition between cognitive and habit memory. Behavioural Brain Research, 193(1), 126-131. https://doi.org/10.1016/j.bbr.2008.05.002

Wolf, O. T. (2008). The influence of stress hormones on emotional memory: relevance for psychopathology. Acta Psychologica, 127(3), 513-531. https://doi.org/10.1016/j.actpsy.2007.08.002

Wolf, O. T. (2009). Stress and memory in humans: twelve years of progress? Brain Research, 1293, 142-154. https://doi.org/10.1016/j.brainres.2009.04.013

Zerbes, G., Kausche, F. M., \& Schwabe, L. (2020). Stress-induced cortisol modulates the control of memory retrieval towards the dorsal striatum. The European Journal of Neuroscience. https://doi.org/10.1111/ejn.14942

Zlomuzica, A., Dere, D., Machulska, A., Adolph, D., Dere, E., \& Margraf, J. (2014). Episodic memories in anxiety disorders: clinical implications. Frontiers in Behavioral Neuroscience, 8 , 131. https://doi.org/10.3389/fnbeh.2014.00131 